\title{
Randomized Controlled Trials of Tianma Gouteng Decoction Combined with Nifedipine in the Treatment of Primary Hypertension: A Systematic Review and Meta-Analysis
}

\author{
Jia Tai, Junbo Zou (D, Xiaofei Zhang, Yu Wang, Yulin Liang, Dongyan Guo, Mei Wang, \\ Chunli Cui, Jing Wang, Jiangxue Cheng, and Yajun Shi
}

\author{
Shaanxi Province Key Laboratory of New Drugs and Chinese Medicine Foundation Research, Pharmacy College, \\ Shaanxi University of Chinese Medicine, Xianyang, 712046, China \\ Correspondence should be addressed to Yajun Shi; 2051004@sntcm.edu.cn
}

Received 24 September 2019; Revised 20 December 2019; Accepted 30 December 2019; Published 7 February 2020

Academic Editor: Jenny M. Wilkinson

Copyright (C) 2020 Jia Tai et al. This is an open access article distributed under the Creative Commons Attribution License, which permits unrestricted use, distribution, and reproduction in any medium, provided the original work is properly cited.

\begin{abstract}
Background. Hypertension is a primary risk factor for cardiovascular disease (CVD). Tianma Gouteng decoction (TGD), originating from Zabingzhengzhixinyi, has been used for thousands of years in China to treat hypertension, giddiness, and migraine. This updated meta-analysis aimed at assessing the efficacy and safety of TGD combined with nifedipine in the treatment of primary hypertension. Methods. Related research published prior to September 1, 2019, was found in electronic databases without language limitations. Fourteen studies were selected and analyzed for specified criteria, including the quality of the studies. All outcomes were recorded exhaustive. Data management and analysis were performed using RevMan 5.3 software. Results. A total of 1,537 (769 cases in the experimental group and 768 cases in the control group) patients were enrolled. The total efficacy rate was improved significantly for the combination of nifedipine with TGD compared to nifedipine treatment alone $\left(I^{2}=22 \%, \mathrm{RR}=1.17\right.$, and $95 \% \mathrm{CI}: 1.12$ to 1.22$)$. Traditional Chinese medicine (TCM) symptoms of patients were obviously improved in the experimental group than in the control group $\left(I^{2}=44 \%, \mathrm{RR}=1.26\right.$, and $95 \% \mathrm{CI}: 1.17$ to 1.36$)$. TGD combined with nifedipine shows a better effect than nifedipine in decreasing diastolic blood pressure $\left(I^{2}=95 \%, \mathrm{MD}=-5.32\right.$, and $95 \% \mathrm{CI}$ : -8.19 to $-2.45)$ and systolic blood pressure $\left(I^{2}=98 \%, \mathrm{MD}=-9.35\right.$, and $95 \% \mathrm{CI}:-15.03$ to -3.67$)$ of patients. A sensitivity analysis was conducted for SBP and DBP by removing 2 studies and recalculated the combined estimate on remaining studies. The results of SBP showed a small heterogeneity $\left(I^{2}=17 \%, \mathrm{MD}=-13.95,95 \% \mathrm{CI}:-14.86\right.$ to -13.05 , and $\left.P<0.00001\right)$ when two studies (shicaihong 2017 and xiaoyugao 2017) were removed. And the results of DBP showed no heterogeneity $\left(I^{2}=0, \mathrm{MD}=-8.36,95 \%\right.$ CI: -8.91 to -7.81 , and $P<0.00001$ ) when two studies (panzhixiong 2019 and shicaihong 2017) were removed. Conclusion. The combination of TGD and nifedipine has a better effect in the treatment of hypertension, including blood pressure lowering and patients' TCMs improving. However, our findings must be handled with care because of the small sample size and low quality of clinic trials cited. Other rigorous and large-scale RCTs are in need to confirm these results.
\end{abstract}

\section{Background}

Hypertension is an important risk factor for cardiovascular disease (CVD) worldwide, and it is also a major risk factor for stroke and coronary heart disease (CHD) in China $[1,2]$. As a calcium channel blocker, nifedipine is employed to broadly treat hypertension in clinics [3]. However, some adverse reactions (AEs) to nifedipine are common, such as edema, rash, headache, and dizziness [4-6]; it can even cause serious adverse reactions within the cardiovascular system, as stated in the drug warnings for nifedipine.

Combination therapy, which is considered to be beneficial for enhancing the antihypertensive effect without increasing AEs [2], is the basic principle for treating hypertension in the Guide to Prevention and Treatment of Hypertension (version 2010). The combination of Chinese and Western medicine is a prevalent therapeutic regimen for treating numerous forms of disease in China. Tianma 
Gouteng decoction (TGD), originating from Zabingzhengzhixinyi, is a classic traditional Chinese medicine (TCM) prescription used for thousands of years in China for the treatment of hypertension, giddiness, and migraine [7, 8]. TGD is composed of Uncaria rhynchophylla (Miq.) Jacks., Gastrodia elata Bl., Scutellaria baicalensis Georgi, Eucommia ulmoides Oliv, Radix cyathulae, Loranthus parasiticus, abalone shell (the abalone shell can be collected without the animal being harmed in any way), Gardenia, Leonurus japonicus, Caulis polygoni multiflori, and Poria cocos, all of which are standard in the Chinese Pharmacopoeia 2015 edition [7].

Several meta-analyses [9-12] have been conducted on the use of TGD to treat primary hypertension focusing on TGD therapy alone. However, the combined use of TCM with Western medicine has been proven to be more effective for many diseases by an increasing number of evidencebased practices. A meta-analysis reported that TGD combined with nifedipine had better effects than nifedipine alone in the treatment of primary hypertension, but the consequence measures were not exactly sufficient [13]. Here, we provided an updated and expanded meta-analysis with timely clinical studies that were mainly conducted between 2014 and 2019. Moreover, the measurements indicated in our paper are more comprehensive than previous reports.

\section{Methods}

2.1. Search Strategy and Selection Criteria. We searched CNKI, PubMed, VIP, EMBASE, Wanfang, Cochrane Library, and CBM. To conduct a comprehensive search, studies published prior to September 1, 2019, were investigated without language limitations. The search terms used were as follows: "Tianma Gouteng decoction" and "hypertension" or "nifedipine" and "hypertension." All corresponding articles were downloaded into Endnote software (version X8, Thomson Reuters, Inc., New York, USA) for further investigation.

2.2. Inclusion and Exclusion Criteria. The inclusion criteria were designed according to the suggestions of doctors, as follows: patients diagnosed as having primary hypertension by meeting the criteria of Guide to Prevention and Treatment of Hypertension 2010, Guiding Principles for Clinical Research of New Drugs in Traditional Chinese Medicine, Chinese Medicine Dialectical Diagnosis Efficacy Standard, Chinese Medicine Diagnosis and Treatment of Heart Disease Efficacy Standards and Norms, or Guide to Prevention and Treatment of Hypertension in China. Studies were presented as randomized control trials (RCTs). The intervention used for patients was TGD combined with nifedipine in the experimental group and only nifedipine in the control group. The measurement of the outcome of each article must have contained a total antihypertensive efficacy. The following indices in the articles must contain at least one of the following: blood pressure, TCMs, serum creatinine, adverse events, and blood urea nitrogen.

The following criteria were utilized to exclude conditions: (1) nonrandomized controlled trials; (2) secondary hypertension; (3) hypertension and other illnesses; (4) patients received drugs other than TGD and nifedipine in RCTs; and (5) studies such as reviews, animal experiments, and case report that were considered to be irrelevant to the theme.

Patients that had systolic blood pressure (SBP) greater than or equal to $140 \mathrm{mmHg}$ and/or diastolic blood pressure (DBP) greater than or equal to $90 \mathrm{mmHg}$ were diagnosed with hypertension in the included studies. To measure the diagnostic efficacy of antihypertension treatment, the total efficiency is equal to significant effect and effective summation. Treatments were considered significantly effective when DBP returned to normal levels and reduced by at least $10 \mathrm{mmHg}$, or DBP did not return to normal levels, but the reduction was at least by $20 \mathrm{mmHg}$. The treatment was considered effective when DBP returned to normal levels and decreased by less than $10 \mathrm{mmHg}$, when DBP did not return to normal levels, but the reduction was by $10 \mathrm{mmHg} 19 \mathrm{mmHg}$, or there was a reduction in systolic blood pressure of at least $30 \mathrm{mmHg}$. Treatment was considered invalid when DBP and SBP did not change significantly or even got worse.

TCM symptoms of hypertension criteria were as follows: (1) significant effect: obvious improvement in clinical symptoms, (2) effective: clinical symptoms slightly improved, and (3) invalid: symptoms and signs have no significant changes or even worse.

2.3. Assessment of Trial Quality. In order to assess the risk of bias, three authors (Jia Tai, Junbo Zou, and Yu Wang) independently evaluated the study validity according to the Cochrane Handbook for Systematic Reviews of Interventions [14].

Six criteria assessing bias and quality were evaluated according to whether the articles described the following:

(1) Random sequence generation (selection bias)

(2) Allocation concealment (selection bias)

(3) Blinding of participants and personnel (performance bias)

(4) Blinding of outcome assessment (detection bias)

(5) Incomplete outcome data (attrition bias)

(6) Selective reporting (reporting bias) and other biases

Three levels were used to assess each checklist item. "Low risk" of bias suggested that the program was sufficient. "High risk" of bias indicated that the description of methods or treatment program was not sufficient enough or was abnormal. "Unclear risk" of bias indicated that there were no descriptions of methods or the treatment program. Any objections among the evaluators (Jia Tai, Junbo Zou, and Yu Wang) were determined through conversation with a fourth author (Yulin Liang).

2.4. Data Extraction. Information from the articles selected in this study included authors, year of publication, number of primary hypertension cases in the experiment and control 
groups, gender and age of patients, treatment period, random method, interventions, and evaluation standard, and the evaluation indexes were independently extracted by the three authors (Jia Tai, Junbo Zou, and Yu Wang). This information is provided and arranged in Tables 1 and 2.

2.5. Statistical Analysis. A Cochrane collaboration metaanalysis review methodology was applied in this study, with Review Manager 5.3 (Cochrane Collaboration) used to perform statistical analysis. The heterogeneity of the studies was determined by $I^{2}$ tests and Q statistics. If the data had low heterogeneity $\left(P \geq 0.1\right.$ and $\left.I^{2} \leq 50 \%\right)$, a fixed-effects model was applied. If the data had high heterogeneity $\left(P<0.1\right.$ or $\left.I^{2}>50 \%\right)$, a random-effects model was applied. Latent issue bias was shown by funnel plots. Index measures, such as antihypertensive efficacy and TCMs, were thought to have dichotomous variables and evaluated by risk ratio (RR) with 95\% confidence intervals (CIs). Continuous variables (such as BP) were rated by the mean difference (MD) with $95 \%$ confidence intervals. The significance of RR or MD was analyzed by a $z$-test, and $P<0.05$ was considered to be indicative of statistical significance. The potential publication bias was assessed by constructing funnel plots.

\section{Results}

3.1. Description of Studies. Studies took place between 2012 and 2017 (Table 1); all were RCTs. A total of 1,733 potentially corresponding studies were identified by our primary search, and 132 articles were exempted for repeat. We excluded 1,514 studies because they obviously did not meet the theme of this paper. Then, a full-text review was conducted on the remaining 87 articles. A total of 73 studies were exempt for the following reasons: 23 studies were animal experiments, 14 articles had vague diagnoses, and 36 articles referred to different intervention methods. Fourteen [15-21, 23-29] studies had adequate index data to permit the calculation of effect sizes for inclusion in this meta-analysis (Figure 1). Of the 14 included studies, 1,537 patients were diagnosed with primary hypertension (769 cases in the experimental group and 768 cases in the control group) and used in this metaanalysis. The intervention used for patients is TGD combined with nifedipine in the experimental group and only nifedipine in the control group. The treatment for primary hypertension in the included studies was slightly different; namely, in the TGD combined with nifedipine treatments, the studies used nifedipine sustained-release tablets or nifedipine controlled-release tablets. In addition, the doses of TGD ranged from approximately 93.4 to $180 \mathrm{~g}$, and the dosage of nifedipine for patients ranged from 20 to $60 \mathrm{mg}$ / day by oral administration (Table 2).

3.2. Quality of Included Trials. All trials were RCTs of participants according to Cochrane risk of bias estimation. The appropriate generation of random distribution sequence was depicted in six [17-19, 24-26] articles. Particular information on distribution was absent from most articles. All studies not used blinding of participants and consequence assessment. Nine [17-21, 23-26] articles had integral outcome data with a low risk of attrition bias. Eight articles [17-19, 21, 23-26] had a low risk of reporting bias as detailed results were given (Figure 2).

3.3. Antihypertensive Efficacy (14 Studies). Effectiveness was defined as an improvement of symptoms. Fourteen articles [15-20, 23-29] reported the total efficacy rate. A fixed-effects model was performed to analyze these studies, and the results showed that TGD combined with nifedipine significantly improved primary hypertension $(\mathrm{RR}=1.17,95 \% \mathrm{CI}$ : 1.12 to 1.22 , and $P<0.00001$; Figure 3 ). There was no statistically significant heterogeneity among the individual trials $\left(P=0.22, \chi^{2}=15.40\right.$, and $\left.I^{2}=22 \%\right)$.

3.4. TCM Improvement (Eight Studies). Eight [16-18, 20, 22, 24, 27] studies measured the improvement of TCMs. There was a statistically significant degree of heterogeneity among individual studies $\left(\chi^{2}=10.65, I^{2}=44 \%\right.$, and $P=$ $0.10)$; therefore, a fixed-effects model was performed for a meta-analysis, which showed that TGD combined with nifedipine can significantly improve TCMs $(\mathrm{RR}=1.26,95 \%$ CI: 1.17 to 1.36 , and $P<0.00001$; Figure 4).

3.5. Decreasing DBP Effect (Eight Studies). Eight [17, 21-28] studies investigated the effectiveness of the combination of TGD and nifedipine in reducing DBP. In the meta-analysis, DBP was significantly reduced $(\mathrm{MD}=-5.32,95 \% \mathrm{CI}:-8.19$ to -2.45 , and $P<0.00001$; Figure 5(a)) with significant heterogeneity among the studies $\left(\chi^{2}=165.74, P=0.0003\right.$, and $\left.I^{2}=95 \%\right)$. TGD combined with nifedipine is preferable to nifedipine in reducing DBP of patients. Due to the large heterogeneity, sensitivity analysis was performed by removing 2 studies and recalculated the combined estimate on remaining studies. And the results of DBP showed no heterogeneity $\left(I^{2}=0, \mathrm{MD}=-8.36,95 \% \mathrm{CI}:-8.91\right.$ to -7.81 , and $P<0.00001$; Figure 5(b)) when two studies (panzhixiong 2019 and shicaihong 2017) were removed.

3.6. Decreasing SBP Effect (Eight Studies). Eight [17, 21-28] trials reported the intervention reflecting SBP. There was heterogeneity among the studies $\left(\chi^{2}=467.63, P<0.00001\right.$, and $\left.I^{2}=98 \%\right)$ and the random-effects model was performed for this analysis. The $\mathrm{MD}$ and $95 \% \mathrm{CI}(\mathrm{MD}=-9.35,95 \% \mathrm{CI}$ : -15.03 to -3.67 , and $P=0.001$; Figure $6(\mathrm{a}))$ indicated a significant decrease of SBP in the experimental group compared with the control group. Sensitivity analysis was performed by removing 2 studies and recalculated the combined estimate on remaining studies due to greater heterogeneity. The result showed a small heterogeneity $\left(I^{2}=17 \%, \quad \mathrm{MD}=-13.95,95 \% \mathrm{CI}: 14.86\right.$ to -13.05 , and $P<0.00001$; Figure 6(b)) when two studies (shicaihong 2017 and xiaoyugao 2017) were removed.

3.7. Serum Creatinine (Scr) and Blood Urea Nitrogen (BUN) (One Study). One trial [17] reported serum creatinine from 
TABLE 1: Basic characteristics of the included trials.

\begin{tabular}{lcccc}
\hline \multirow{2}{*}{ Study } & Patients $(n)$ & \multicolumn{2}{c}{ Sex (male/female) } & \\
& Experimental group/control group & Experimental group & Control group & Diagnostic standard \\
\hline Peifeng, 2015 [15] & $33 / 33$ & $21 / 12$ & C:21/12 & NR \\
Xiaofei et al., 2016 [16] & $40 / 40$ & $22 / 18$ & $20 / 20$ & GPTH2010 and GPCREDTCM \\
Tao, 2016 [17] & $61 / 61$ & $22 / 18$ & $20 / 20$ & GPTH2010 and CMDDES \\
Yingke, 2014 [18] & $60 / 60$ & $32 / 28$ & $33 / 27$ & GPCREDTCM \\
Jinbo, 2015 [19] & $154 / 153$ & $87 / 67$ & $75 / 78$ & NR \\
Fan and Lili, 2015 [20] & $30 / 30$ & NR & NR & CMDTHDESN \\
Pan, 2019 [21] & $52 / 52$ & $26 / 26$ & $25 / 27$ & NR \\
Xiaofang, 2016 [22] & $55 / 55$ & $30 / 25$ & $32 / 23$ & GPTH2010 \\
Fu, 2018 [23] & $40 / 40$ & $22 / 18$ & $24 / 16$ & GPTH2010 \\
Caihong, 2017 [24] & $40 / 40$ & $5 / 15$ & $23 / 17$ & GPTH2010 and GPCREDTCM \\
Dai, 2019 [25] & $55 / 55$ & NR & NR & NR \\
Yugao, 2017 [26] & $23 / 23$ & $25 / 21$ & $13 / 10$ & GPCREDTCM \\
Peng et al., 2016 [27] & $46 / 46$ & $48 / 32$ & $26 / 20$ & NR \\
Yingjun, 2015 [28] & $80 / 80$ & $46 / 34$ & GPTH2010 and GPCREDTCM \\
\hline
\end{tabular}

Note: NR: no report; GPTH2010: Guide to Prevention and Treatment of Hypertension 2010; GPCREDTCM: Guiding Principles for Clinical Research of New Drugs in Traditional Chinese Medicine; CMDDES: Chinese Medicine Dialectical Diagnosis Efficacy Standard; CMDTHDESN: Chinese Medicine Diagnosis and Treatment of Heart Disease Efficacy Standards and Norms; GPTHC: Guide to Prevention and Treatment of Hypertension in China (trial version).

TABLE 2: Intervention characteristics of the included trials.

\begin{tabular}{|c|c|c|c|c|}
\hline \multirow{2}{*}{ Study } & \multicolumn{2}{|r|}{ Dosage } & \multirow{2}{*}{ Duration } & \multirow{2}{*}{ Outcome } \\
\hline & Control group & Trial group & & \\
\hline $\begin{array}{l}\text { Peifeng, } 2015 \\
{[15]}\end{array}$ & $\mathrm{NF}$ (2 times/d, $30 \mathrm{mg} /$ times) & $\begin{array}{c}\text { TGD }(52.5 \mathrm{~g} / \text { times, } 2 \sim 3 \text { times } / \mathrm{d})+\mathrm{NF}(2 \text { times } / \mathrm{d}, \\
30 \mathrm{mg} / \mathrm{times})\end{array}$ & 1 month & Ae \\
\hline $\begin{array}{l}\text { Xiaofei et al., } \\
2016 \text { [16] }\end{array}$ & NF (1 times/d, $30 \mathrm{mg} /$ times $)$ & $\begin{array}{c}\text { TGD }(51.2 \mathrm{~g} / \text { times, } 2 \sim 3 \text { times } / \mathrm{d})+\mathrm{NF}(1 \text { times } / \mathrm{d} \text {, } \\
30 \mathrm{mg} / \mathrm{times})\end{array}$ & 2 weeks & Ae TCMS \\
\hline Tao, 2016 [17] & $\mathrm{NF}$ (2 times/d, $10 \mathrm{mg} /$ times $)$ & $\begin{array}{c}\text { TGD }(48.4 \mathrm{~g} / \text { times, } 2 \sim 3 \text { times } / \mathrm{d})+\mathrm{NF}(2 \text { times } / \mathrm{d}, \\
10 \mathrm{mg} / \mathrm{times})\end{array}$ & 8 weeks & BP Scr BUN \\
\hline $\begin{array}{l}\text { Yingke, } 2014 \\
{[18]}\end{array}$ & NFS (2 times/d, $10 \mathrm{mg} /$ times) & $\begin{array}{c}\text { TGD (52 g/times, } 2 \sim 3 \text { times } / \mathrm{d})+\mathrm{NFS}(2 \text { times } / \mathrm{d} \text {, } \\
10 \mathrm{mg} / \text { times })\end{array}$ & 2 weeks & Ae TCMS \\
\hline Jinbo, 2015 [19] & NFS (2 times/d, $10 \mathrm{mg} /$ times) & TGD (57 g/times, 2 times/d) + NFS ( 2 times $/ \mathrm{d}, 10 \mathrm{mg} /$ & 2 weeks & $\mathrm{Ae}$ \\
\hline $\begin{array}{l}\text { Fan and Lili, } \\
2015[20]\end{array}$ & NFS ( 1 times/d, $5 \mathrm{mg} /$ times) & TGD (87.5 g/times 2 times $/ \mathrm{d})+\mathrm{NFS}(1$ times $/ \mathrm{d}, 5 \mathrm{mg} /$ & 3 months & $\mathrm{Ae}$ \\
\hline Pan, 2019 [21] & NFS (1 times/d, $20 \mathrm{mg} /$ times) & $\begin{array}{c}\text { TGD (90 g/times, } 2 \text { times } / \mathrm{d})+ \text { NFS }(1 \text { times } / \mathrm{d}, 20 \mathrm{mg} / \\
\text { times })\end{array}$ & 2 weeks & $\mathrm{BP}$ \\
\hline $\begin{array}{l}\text { Xiaofang, } 2016 \\
{[22]}\end{array}$ & NFS (2 times/d, $20 \mathrm{mg} /$ times) & TGD $(125 \mathrm{~g} / \mathrm{d})+\mathrm{NFS}(2$ times $/ \mathrm{d}, 20 \mathrm{mg} /$ times $)$ & NR & Ae BP Ar \\
\hline $\mathrm{Fu}, 2018[23]$ & NFS (2 times/d, $20 \mathrm{mg} /$ times) & $\begin{array}{c}\text { TGD ( } 57 \mathrm{~g} / \text { times, } 2 \text { times } / \mathrm{d})+\mathrm{NFS} \text { ( } 2 \text { times } / \mathrm{d}, 20 \mathrm{mg} / \\
\text { times })\end{array}$ & 6 months & Ae BP Ar \\
\hline $\begin{array}{l}\text { Caihong, } 2017 \\
\text { [24] }\end{array}$ & NFS (1 times/d, 30 mg/times) & $\begin{array}{c}\text { TGD (79 g/times, } 2 \text { times } / \mathrm{d})+\mathrm{NFS}(1 \text { times } / \mathrm{d}, 30 \mathrm{mg} / \\
\text { times })\end{array}$ & NR & Ae BP \\
\hline Dai, 2019 [25] & NF (1 times/d, $20 \mathrm{mg} /$ times) & TGD $(\mathrm{NR})+\mathrm{NF}(1$ times $/ \mathrm{d}, 20 \mathrm{mg} /$ times $)$ & 2 weeks & $\mathrm{Ae} \mathrm{BP} \mathrm{Ar}$ \\
\hline $\begin{array}{l}\text { Yugao, } 2017 \\
{[26]}\end{array}$ & NFS (2 times/d, $10 \mathrm{mg} /$ times) & $\begin{array}{c}\text { TGD ( } 52 \mathrm{~g} / \text { times, } 3 \text { times } / \mathrm{d})+ \text { NFS }(2 \text { times } / \mathrm{d}, 10 \mathrm{mg} / \\
\text { times })\end{array}$ & 8 weeks & $\begin{array}{l}\text { Ae BP life } \\
\text { quality }\end{array}$ \\
\hline $\begin{array}{l}\text { Peng et al., } 2016 \\
\text { [27] }\end{array}$ & $\begin{array}{l}\text { NFC ( } 1 \text { times } / \mathrm{d}, 30 \mathrm{mg} / \text { times, after } \\
\text { half a month, } 2 \text { times } / \mathrm{d}, 30 \mathrm{mg} / \text { times })\end{array}$ & $\begin{array}{c}\text { TGD ( } 46.7 \mathrm{~g} / \text { times } 2 \text { times/d) }+ \text { NFC }(1 \text { times } / \mathrm{d} \text {, } \\
30 \mathrm{mg} / \mathrm{times} \text {, after half a month, } 2 \text { times } / \mathrm{d}, 30 \mathrm{mg} / \\
\text { times })\end{array}$ & 2 months & Ae BP \\
\hline $\begin{array}{l}\text { Yingjun, } 2015 \\
{[28]}\end{array}$ & NFS (2 times/d, $10 \mathrm{mg} /$ times) & $\begin{array}{c}\text { TGD (68.8 g/times, } 3 \text { times } / \mathrm{d})+ \text { NFS }(2 \text { times } / \mathrm{d} \text {, } \\
10 \mathrm{mg} / \mathrm{times})\end{array}$ & NR & Ae BP \\
\hline
\end{tabular}

Notes: NF: nifedipine; NFS: nifedipine sustained-release tablets; NFC: nifedipine controlled-release tablets; NR: no report; Ae: antihypertensive effect; TCMS: traditional Chinese medicine symptoms; BP: blood pressure; Scr: serum creatinine; BUN: blood urea nitrogen; Ar: adverse reactions. TGD was taken as a decoction when taken by patients.

$90.82 \pm 9.47 \mu \mathrm{mol} / \mathrm{L}$ to $70.46 \pm 7.51 \mu \mathrm{mol} / \mathrm{L}$ and blood urea nitrogen from $6.91 \pm 1.28 \mathrm{mmol} / \mathrm{L}$ to $4.17 \pm 1.02 \mathrm{mmol} / \mathrm{L}$ after treatment in the experimental group. In the control group, the level of serum creatinine reduced from
$91.76 \pm 10.73 \mu \mathrm{mol} / \mathrm{L}$ to $83.15 \pm 8.92 \mu \mathrm{mol} / \mathrm{L}$ and blood urea nitrogen decreased from $6.73 \pm 1.35 \mathrm{mmol} / \mathrm{L}$ to $5.38 \pm 1.87 \mathrm{mmol} / \mathrm{L}$. Compared to the control group, the Scr and BUN level of the experimental group showed a greater 


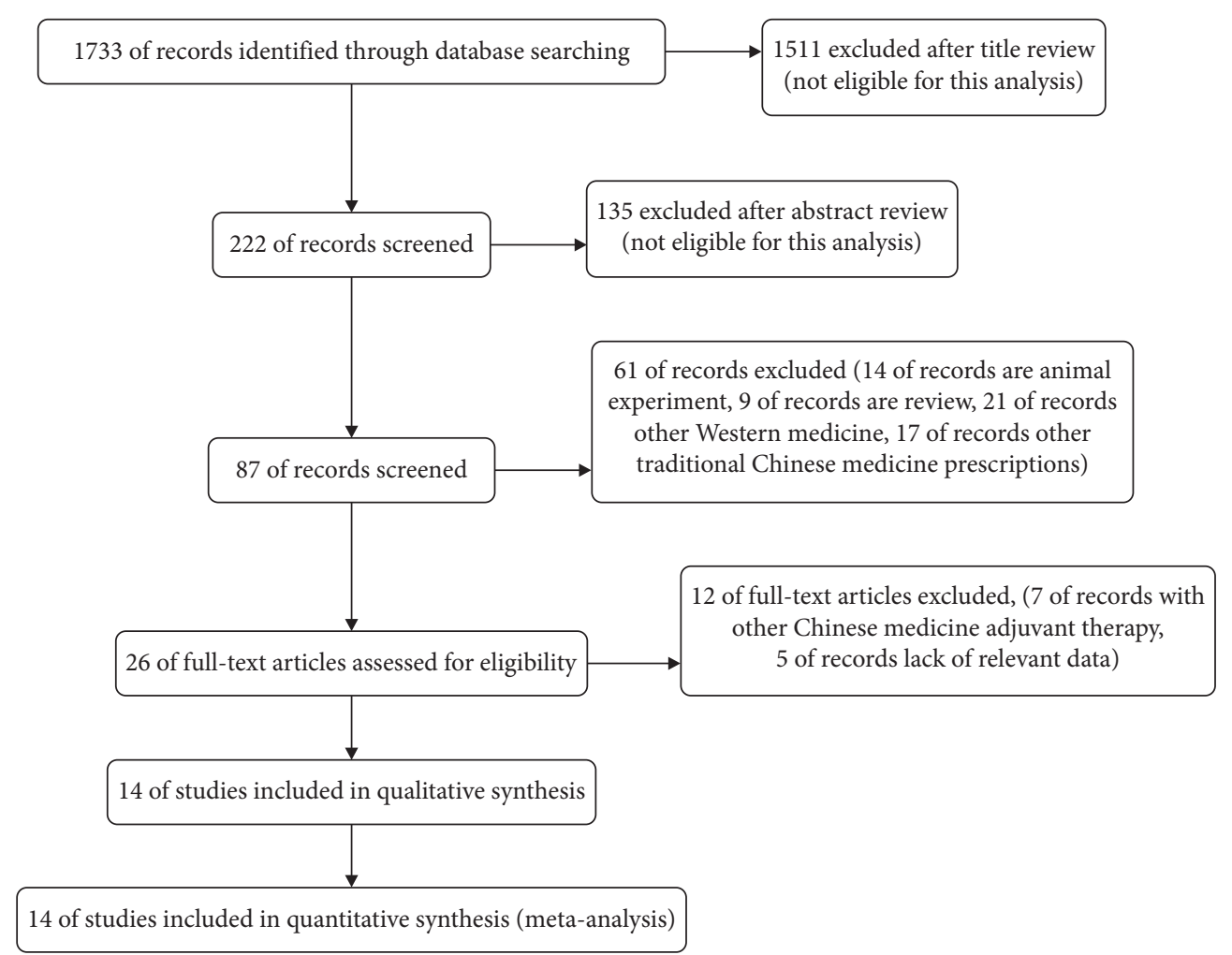

FIgURe 1: Processing of the studies extracted for the meta-analysis.

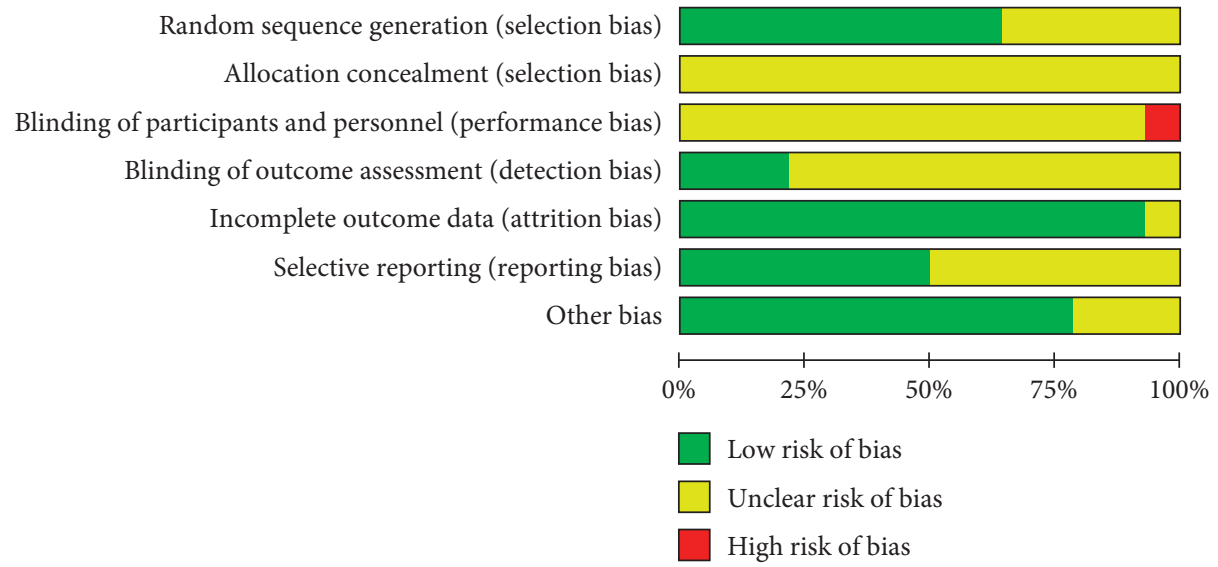

Figure 2: Risk of bias assessment in eligible studies. The quality assessment was conducted by Review Manager 5.3 according to the Cochrane Handbook for Systematic Reviews of Interventions version 5.1.0. Red, high risk of bias; green, low risk of bias; yellow, unclear risk of bias.

decrease. However, the small sample size prevents any firm conclusions from being inferred.

3.8. AEs (Two Studies). Four trials [22-25] provided descriptions on AEs such as dizziness, gastrointestinal reaction, stomach discomfort, diarrhea, and ankle edema. Nevertheless, these symptoms disappeared without treatment.

3.9. Publication Bias. Publication bias was conducted by a funnel plot. In this study, funnel plots were conducted of
TGD combined with nifedipine vs. nifedipine alone on antihypertensive efficacy, TCMs, and BP. Except for Figure 7 , other funnel plots were usually symmetrical, which indicated no evident publication bias (Figures 7-10). Although we conducted comprehensive searches, we identified and included 14 trials; all of them were conducted and published in Chinese. All of the trials had small sample sizes. We tried to avoid language bias and location bias, but we could not exclude potential dissemination bias. Study publications provided only limited descriptions of study design, allocation concealment, and baseline data. All of the 


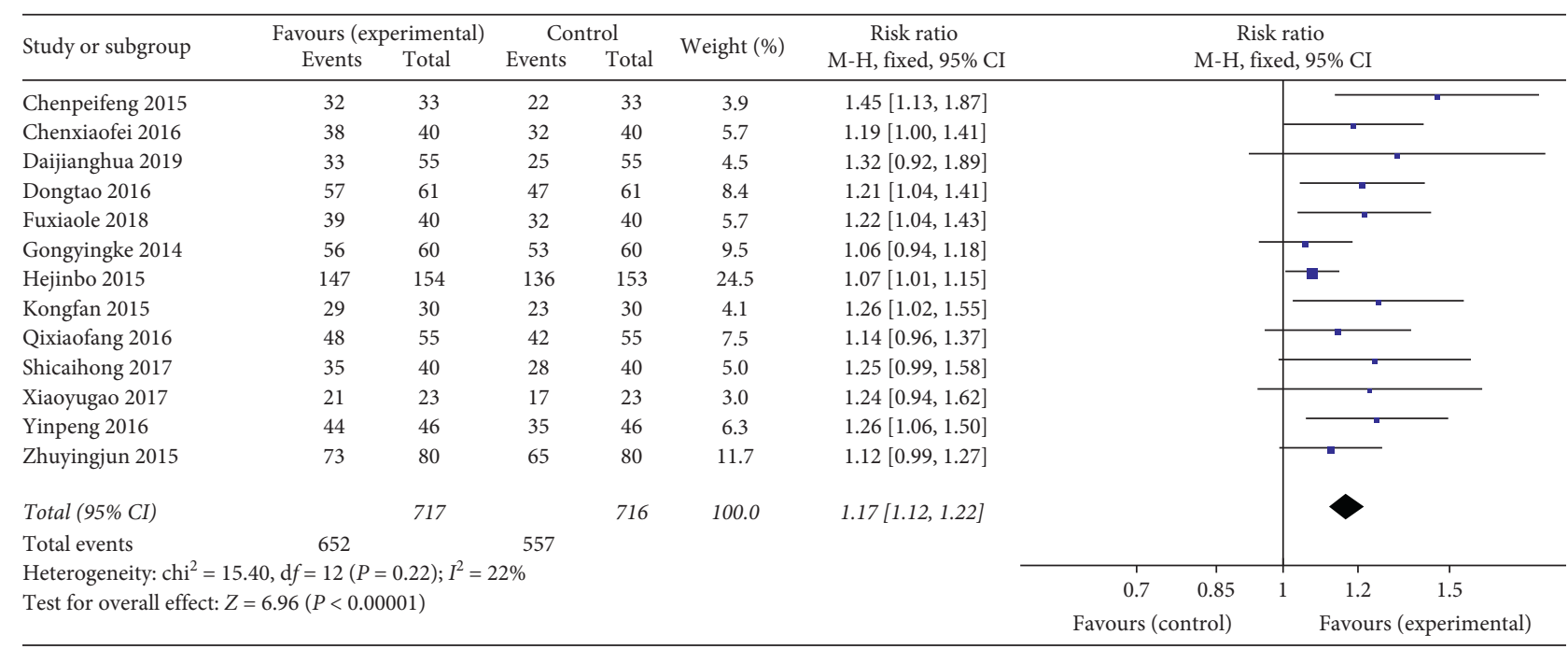

Figure 3: Forest plot of antihypertensive effect. Note: experiment: Tianma Gouteng decoction combined with nifedipine; control: nifedipine.

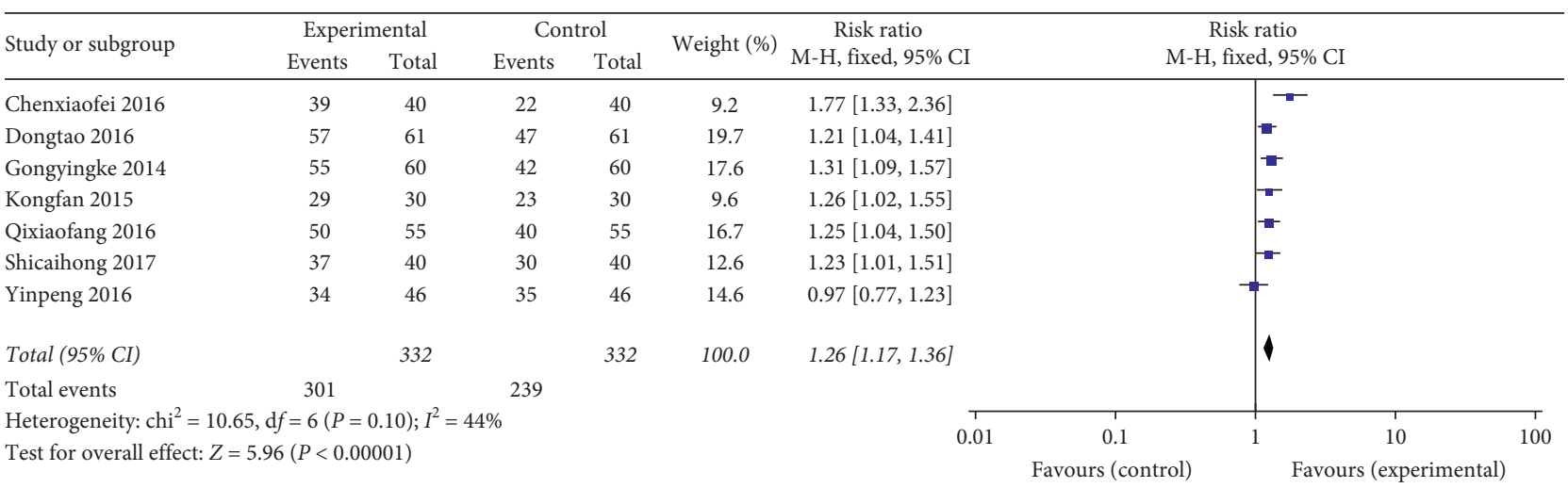

Figure 4: Forest plot of the improvement of traditional Chinese medicine symptoms. Note: experiment: Tianma Gouteng decoction combined with nifedipine; control: nifedipine.

RCTs included in this review showed a mostly unclear risk of bias in more than one "risk of bias" domains.

\section{Discussion}

As the first inducer for CVD, hypertension will be the number one "killer" of human beings by 2020 [30, 31]. The overall prevalence of hypertension in adults is approximately $30-45 \%$ [32], and a SBP of $20 \mathrm{mmHg}$ higher than average and DBP $10 \mathrm{mmHg}$ higher than average were each associated with a doubling in the risk of death from stroke, heart disease, or other vascular diseases [33]. In a World Health Organization report, the number of hypertensive patients increased from 600 million in 1980 to 1 billion in 2008 [30], and this number is still rising. However, if hypertension is controlled, CVD events will significantly decrease. In the United Kingdom, the incidence of stroke is forecasted to drop by $28 \%-44 \%$, and the incidence of ischemic heart disease will reduce by $20-35 \%$ through the control hypertension [34]. A report from the American College of
Cardiology/American Heart Association indicated that there are more potentially preventable CVD events attributable to elevated BP in individuals with higher than with lower risk of CVD [33]. Therefore, treating hypertension is critical for protecting against the occurrence of CVD.

Nifedipine is commonly used to treat hypertension in clinical practice, but it has some limitations [35-39]. In the theory of TCM, the mechanism of primary hypertension pertains to dizziness, headache, hyper-yang of liver, and insufficiency of liver-yin [40]. TGD can treat hyper-yang of liver, as well as the upward disturbance of liver wind [7]. Although there are some limitations for TCM due to the shortage of sufficient studies, increasingly valid evidencebased practice makes it an attractive therapy system for various diseases. Numerous recent studies have found that TGD can affect the renin-angiotensin-aldosterone system to reduce angiotensin and plasma endothelium levels that can influence BP [41]. Sixty-seven hypothalamic protein expressions increased more than two times, and 19 hypothalamic protein expressions decreased more than two times 


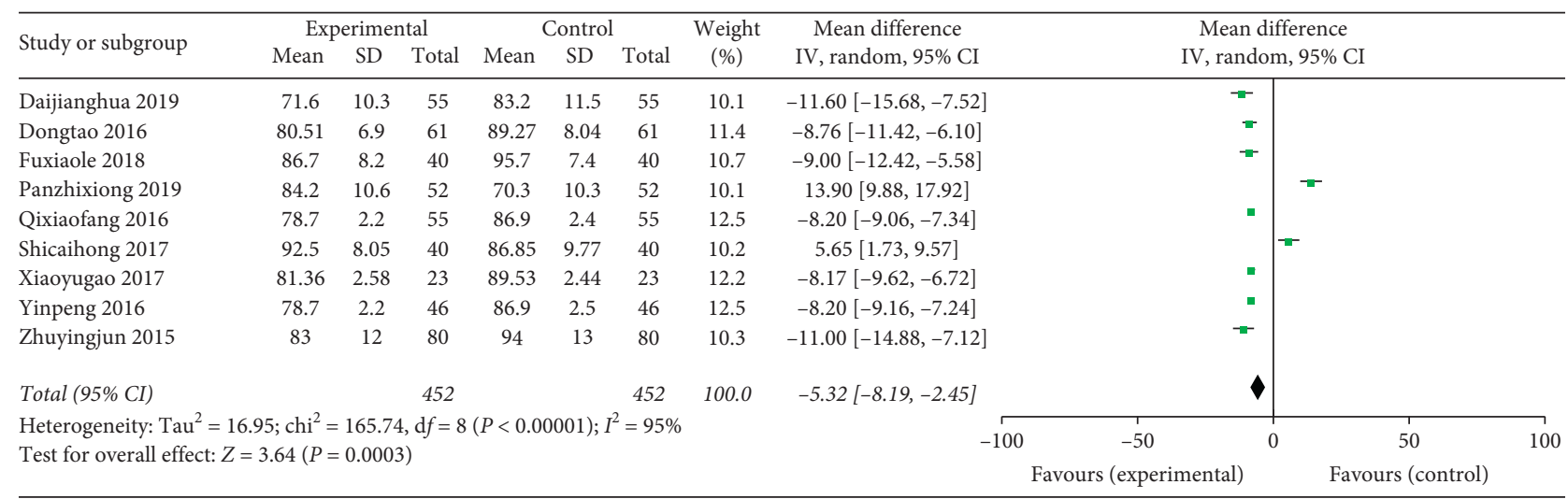

(a)

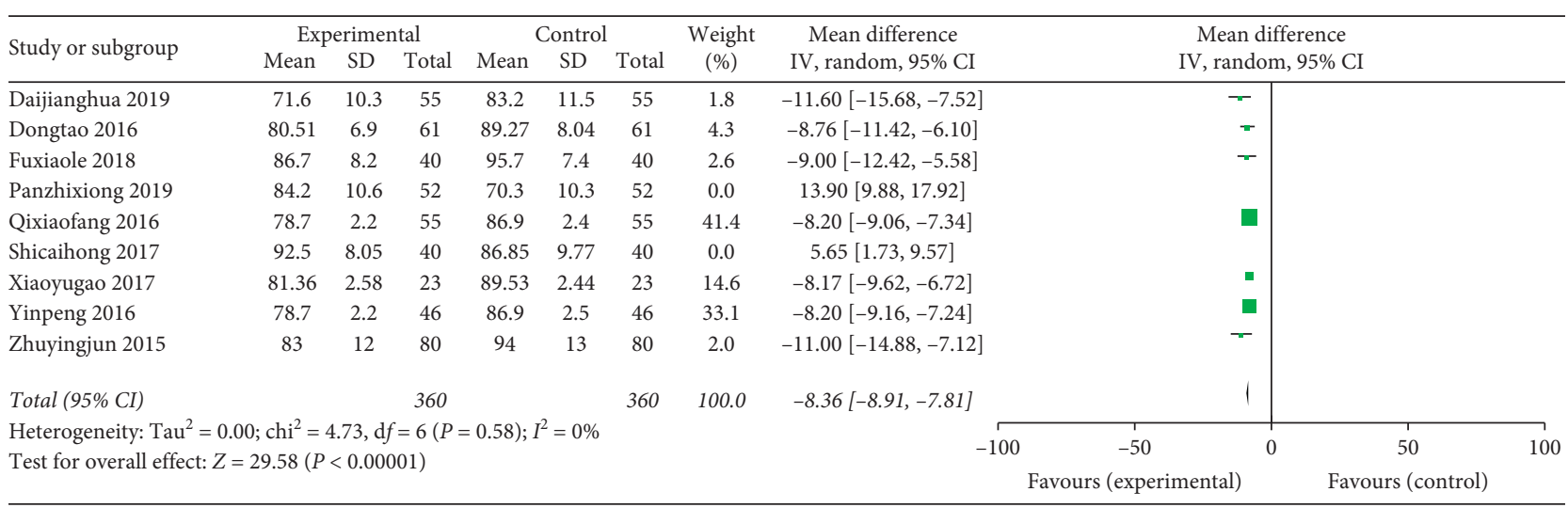

(b)

FiguRE 5: Forest plot of decreasing diastolic blood pressure effect. Note: experiment: Tianma Gouteng decoction combined with nifedipine; control: nifedipine. (a) No sensitivity analysis and (b) after sensitivity analysis.

after taking TGD. These changes may be related to the mechanism of TGD in treating hypertension [42]. TGD can improve endothelial function in patients with hypertension via the mechanism of increasing GCH-PX and CAT content, remove excess oxygen free radicals, and prevent lipid peroxidation of vascular endothelial cells [43]. TGD can reduce vasoconstrictor substances in hypertensive patients and protect and regulate vascular endothelium secretory function [44]. Moreover, as a primary component of TGD prescription, Gastrodia elata plays an important role in regulating the flow of coronary blood in patients [45]. Gastrodia elata could perform its vasodilator effect not only by inhibiting vascular smooth muscle contraction, but also by enhancing blood vessel elasticity and stabilizing the arterial structure [46]. Gastrodia elata can antagonize epinephrine, regulate blood vessels, and dilate small blood vessels, thereby regulating blood pressure. In addition, several clinical studies found that Uncaria could decrease BP effectively by regulating the above biomarkers and metabolic pathways [47]. The antihypertensive effect of Uncaria alkaloids is related to the decrease in frequency [48].

In clinical studies, total efficiency is a measure for judging the antihypertensive efficacy of drugs. As the results of this meta-analysis showed, the total effective rate for the treatment of hypertension in the experimental group was
90.93\% (652/717), which was higher than the control group (77.79\% (557/716)). Compared with the control group, the experimental group showed better in antihypertension effect $(\mathrm{RR}=1.17,95 \% \mathrm{CI}: 1.12$ to 1.22 , and $P<0.00001)$. Hypertension in patients is also accompanied by dizziness, headache, tinnitus, and insomnia [40]. However, these symptoms significantly improved in the experimental group $(\mathrm{RR}=1.26,95 \% \mathrm{CI}=1.17$ to 1.36 , and $P<0.00001)$. The BP level also reduced in the experimental group more than the control group (DBP : $\mathrm{MD}=-5.32,95 \% \mathrm{CI}:-8.19$ to -2.45 , and $P<0.00001 ; \mathrm{SBP}: \mathrm{MD}=-9.35,95 \% \mathrm{CI}: 15.03$ to -3.67 , and $P=0.001)$. Only two studies reported AEs of TGD combined with nifedipine.

However, substantial heterogeneity was detected between included studies when we studied SBP and DBP outcomes. First, a sensitivity analysis was conducted, in which 1 study at a time was removed and the individual study would not have a significant impact on the results. Second, we conducted a sensitivity analysis by removing 2 studies and recalculated the combined estimate on remaining studies. The results of SBP showed a small heterogeneity $\left(I^{2}=17 \%, \mathrm{MD}=-13.95,95 \% \mathrm{CI}: 14.86\right.$ to -13.05 , and $P<0.00001$ ) when two studies (shicaihong 2017 and xiaoyugao 2017) were removed. And the results of DBP showed no heterogeneity $\left(I^{2}=0, \mathrm{MD}=-8.36,95 \% \mathrm{CI}:-8.91\right.$ 


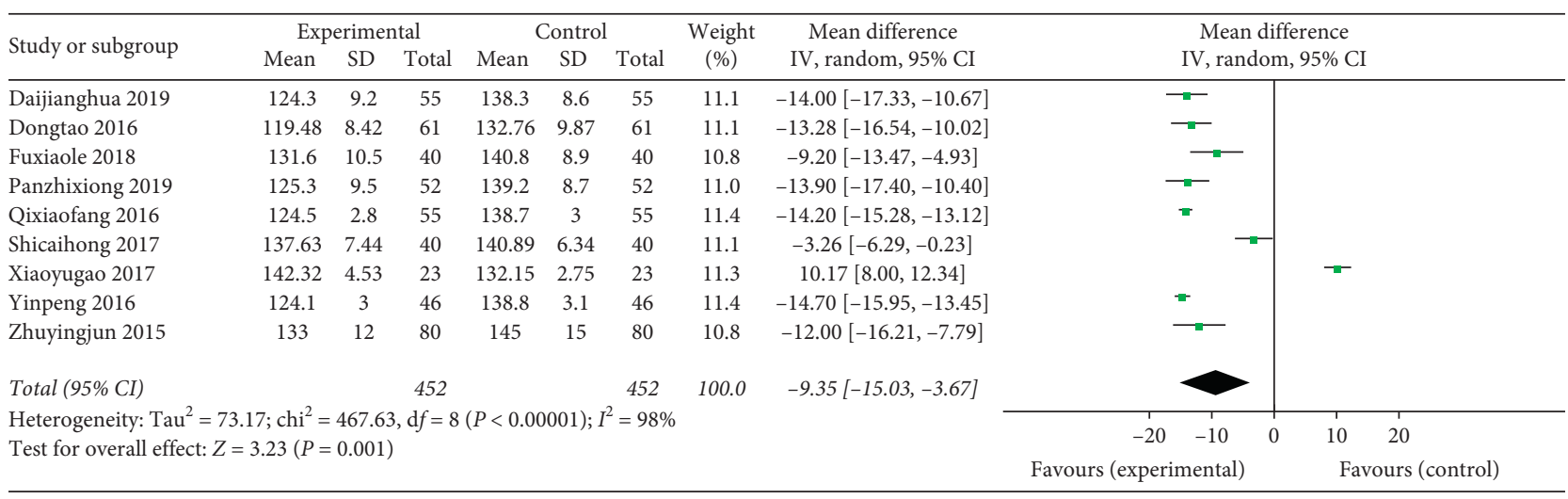

(a)

\begin{tabular}{|c|c|c|c|c|c|c|c|c|c|c|c|}
\hline \multirow{3}{*}{$\begin{array}{l}\text { Study or subgroup } \\
\text { Daijianghua } 2019\end{array}$} & \multicolumn{3}{|c|}{ Experimental } & \multicolumn{3}{|c|}{ Control } & \multirow{2}{*}{$\begin{array}{l}\text { Weight } \\
(\%)\end{array}$} & \multirow{2}{*}{$\begin{array}{l}\text { Mean difference } \\
\text { IV, random, 95\% CI }\end{array}$} & \multirow{2}{*}{\multicolumn{3}{|c|}{$\begin{array}{l}\text { Mean difference } \\
\text { IV, random, } 95 \% \text { CI }\end{array}$}} \\
\hline & Mean & SD & Total & Mean & $\mathrm{SD}$ & Total & & & & & \\
\hline & 124.3 & 9.2 & 55 & 138.3 & 8.6 & 55 & 6.8 & $-14.00[-17.33,-10.67]$ & 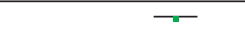 & & \\
\hline Dongtao 2016 & 119.48 & 8.42 & 61 & 132.76 & 9.87 & 61 & 7.1 & $-13.28[-16.54,-10.02]$ & $\longrightarrow$ & & \\
\hline Fuxiaole 2018 & 131.6 & 10.5 & 40 & 140.8 & 8.9 & 40 & 4.3 & $-9.20[-13.47,-4.93]$ & & & \\
\hline Panzhixiong 2019 & 125.3 & 9.5 & 52 & 139.2 & 8.7 & 52 & 6.2 & $-13.90[-17.40,-10.40]$ & 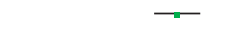 & & \\
\hline Qixiaofang 2016 & 124.5 & 2.8 & 55 & 138.7 & 3 & 55 & 38.4 & $-14.20[-15.28,-13.12]$ & $\mathbf{\square}$ & & \\
\hline Shicaihong 2017 & 137.63 & 7.44 & 40 & 140.89 & 6.34 & 40 & 0.0 & $-3.26[-6.29,-0.23]$ & & & \\
\hline Xiaoyugao 2017 & 142.32 & 4.53 & 23 & 132.15 & 2.75 & 23 & 0.0 & $10.17[8.00,12.34]$ & & & \\
\hline Yinpeng 2016 & 124.1 & 3 & 46 & 138.8 & 3.1 & 46 & 32.7 & $-14.70[-15.95,-13.45]$ & $=$ & & \\
\hline Zhuyingjun 2015 & 133 & 12 & 80 & 145 & 15 & 80 & 4.4 & $-12.00[-16.21,-7.79]$ & $\longrightarrow$ & & \\
\hline Total (95\% CI) & & & 389 & & & 389 & 100.0 & $-13.95[-14.86,-13.05]$ & $\downarrow$ & & \\
\hline \multirow{2}{*}{\multicolumn{9}{|c|}{$\begin{array}{l}\text { Heterogeneity: } \mathrm{Tau}^{2}=0.25 ; \mathrm{chi}^{2}=7.21, \mathrm{~d} f=6(P=0.30) ; I^{2}=17 \% \\
\text { Test for overall effect: } Z=30.13(P<0.00001)\end{array}$}} & $-20 \quad-10$ & 10 & 20 \\
\hline & & & & & & & & & Favours (experimental) & Fav & ours (control) \\
\hline
\end{tabular}

(b)

Figure 6: Forest plot for decreasing systolic blood pressure effect. Note: experiment: Tianma Gouteng decoction combined with nifedipine; control: nifedipine. (a) No sensitivity analysis and (b) after sensitivity analysis.

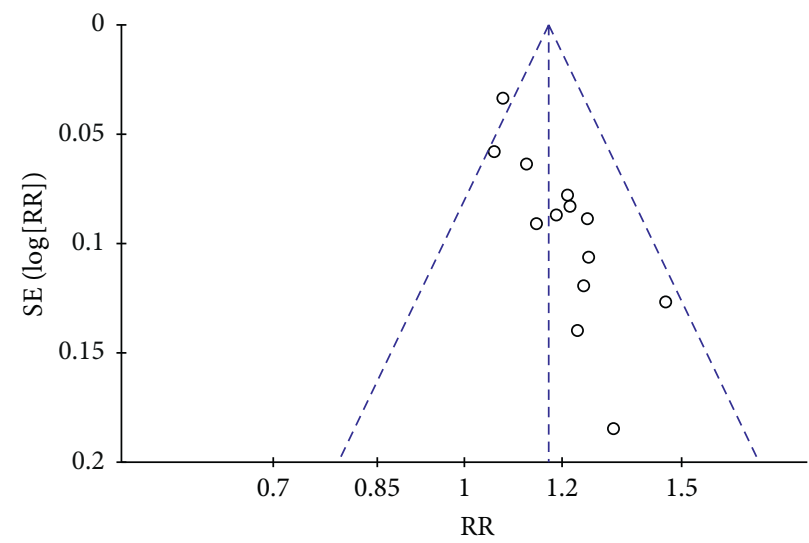

Figure 7: Funnel plot of antihypertensive efficacy for the publication bias.

to -7.81 , and $P<0.00001$ ) when two studies (panzhixiong 2019 and shicaihong 2017) were removed. We made a detailed analysis of the included literature and found that there were significant differences in the sex ratio of patients in the experimental group in the shicaihong 2017 and xiaoyugao 2017 studies. Study publications provided only limited descriptions of study design, allocation concealment, and baseline data, and there are a few indicators of measurement. All of the RCTs included in this review showed a mostly unclear risk of bias in more than one "risk of bias" domains. These reasons may lead to poor heterogeneity in the research process, as well as funnel diagram asymmetry. It is suggested that international standards should be used in clinical research to improve the quality of methodology and strengthen the quality of research and optimization methodology. At the same time, the details such as the generation of random sequence, the concealment of distribution, and the implementation of random allocation should be clarified 


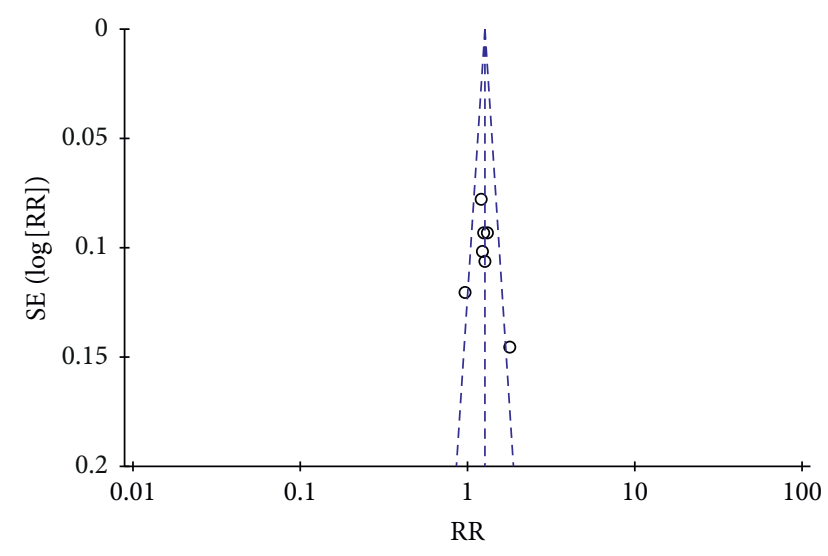

FIgURE 8: Funnel plot of traditional Chinese medicine symptoms for the publication bias.

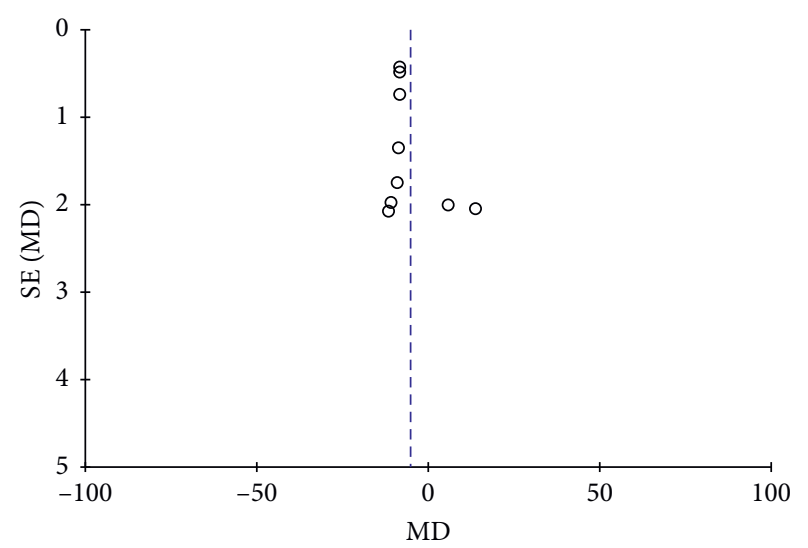

Figure 9: Funnel plot of diastolic blood pressure for the publication bias.

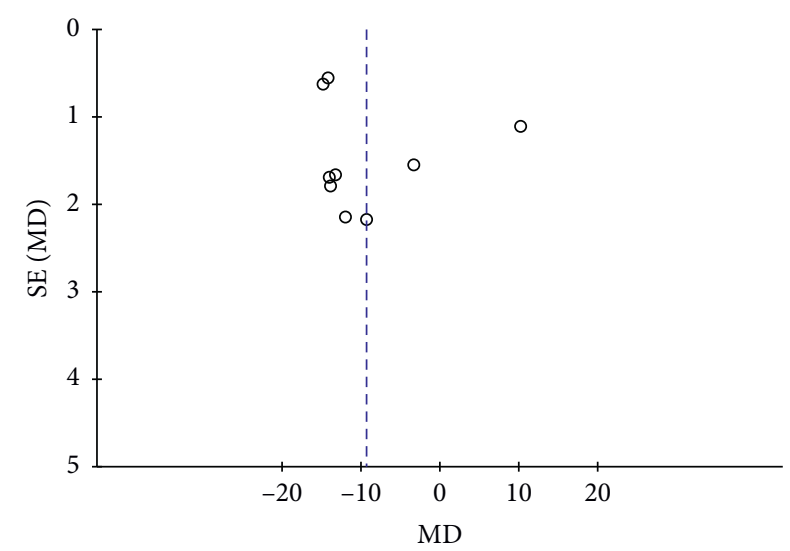

FIGURE 10: Funnel plot of systolic blood pressure for the publication bias.

in the research report. In addition, it should also develop in the direction of international cooperation, multicenter, large sample, complex random grouping, and so on. In addition, there are limitations to this research, such as the low quality of eligible trials, the lack of strict methodologies, and the employment of sole race rather than a more varied population sample. It is necessary to examine the results using other rigorous and large-scale RCTs.

\section{Conclusion}

According to the results and conclusions of the article, we can see that the combination of TGD and nifedipine has a better effect in the treatment of hypertension, so we suggest that we can adopt the method of combination of traditional Chinese and Western medicine according to the patient's condition. However, our findings must be handled with care because of the small sample size and low quality of clinic trials cited. Other rigorous and large-scale RCTs are in need to confirm these results.

\section{Abbreviations:}

CVD: Cardiovascular disease

TGD: Tianma Gouteng decoction

RCTs: Randomized controlled trials

BP: $\quad$ Blood pressure

DBP: Diastolic blood pressure

SBP: $\quad$ Systolic blood pressure

TCMs: Traditional Chinese medicine symptoms

RR: Risk ratio

OR: Odds ratio

MD: Mean difference

CI: $\quad$ Confidence intervals

Scr: $\quad$ Serum creatinine

BUN: Blood urea nitrogen.

\section{Data Availability}

All data generated or analyzed during this study are included in this article.

\section{Conflicts of Interest}

The authors declare that they have no conflicts of interest.

\section{Authors' Contributions}

JT, JZ, and XZ contributed equally to this work. JT, JBZ, and YW searched articles in electronic databases and wrote the manuscript. YLL, XFZ, DYG, and MW analyzed the data. CCHC, JW, and JXC performed data extraction. YJS designed the study and revised the paper.

\section{Acknowledgments}

This project was supported by the Natural Science Foundation of China (grant number: 81703720) (support to XFZ), Key Research and Development Program of Shaanxi Province, China (grant number: 2017ZDXM-SF-008) (support to YJS), Chinese Medicine Pharmaceutical Key Discipline of Shaanxi Province (grant number: 303061107) (support to YJS), Key Research and Development Plan of Shaanxi Province (grant number: 2018SF-314) (support to YJS), and Discipline Innovation team Project of Shaanxi 
University of Chinese Medicine (2019-YL11) (support to YJS). The authors would like to acknowledge the Shaanxi Province Key Subject of Pharmacy Engineering of Shaanxi Provincial Traditional Chinese Medicine Administration.

\section{References}

[1] I. Hajjar and T. Kotchen, "Trends in prevalence, awareness, treatment, and control of hypertension in the United States, 1988-2000," JAMA, vol. 290, no. 2, p. 199, 2003.

[2] L. Lisheng, "China hypertension prevention guide 2010," Chinese Journal of Hypertension, vol. 10, no. 8, pp. 701-743, 2011.

[3] T. Linian, W. Fang, and C Gang, "Adverse reactions of nifedipine,” Straits Pharmaceutical, vol. 2, pp. 266-268, 2013.

[4] J. Wenyan and Y. Song, "Analysis of the efficacy and drug understanding of nifedipine sustained-release preparation in the treatment of hypertension," Journal of Clinical Medical Literature, vol. 5, no. 37, p. 163, 2018.

[5] L. Jingzhe, "Effect of nifedipine sustained release tablets and nifedipine controlled release tablets in the treatment of essential hypertension," Guide of China Medicine, vol. 16, no. 27, pp. 160-161, 2018.

[6] S. Chan, "Clinical observation of amlodipine and nifedipine in the treatment of hypertension," Chinese Journal of Urban and Rural Enterprise Hygiene, vol. 33, no. 4, pp. 130-132, 2018.

[7] L. Ji, Pharmacology of Traditional Chinese Medical Formulae: Beijing: China Traditional Chinese Medicine, Publishing House, Manhattan, USA, 2012.

[8] J. Hq and L. Yl, "Urine metabonomic study on hypertension patients of ascendant hyperactivity of gan yang syndrome by high performance liquid chromatography coupled with time of flight mass spectrometry," Chinese Journal of Integrated Traditional and Western Medicine, vol. 32, no. 3, pp. 333-337, 2012.

[9] B. Chen, Y. Wang, Z. He, D. Wang, X. Yan, and P. Xie, "Tianma gouteng decoction for essential hypertension: protocol for a systematic review and meta-analysis," Medicine (Baltimore), vol. 97, no. 8, 2018.

[10] J. Wang, B. Feng, X. Yang et al., “Tianma gouteng yin as adjunctive treatment for essential hypertension: a systematic review of randomized controlled trials," Evidence-Based Complementary and Alternative Medicine, vol. 2013, 2013.

[11] X. Xiong, P. Wang, Y. Zhang, and X. Li, "Effects of traditional chinese patent medicine on essential hypertension," Medicine, vol. 94, no. 5, p. e442, 2015.

[12] Z. Jb and Z. Xf, “The therapeutic efficacy of danhong injection combined with percutaneous coronary intervention in acute coronary syndrome: a systematic review and meta-analysis," Frontiers in Pharmacology, vol. 9, p. 550, 2018.

[13] L. Bingqing, G. Hongyu, W. Xiaofang, and X. Shaoxiang, "Systemic and meta-analysis of tianma gouteng decoction combined with nifedipine in the treatment of essential hypertension," Inner Mongolia Traditional Chinese Medicine, vol. 12, pp. 52-54, 2016.

[14] J. J. Deeks, J. P. T. Higgins, D. G. Altman, and S. Green, "Cochrane handbook for systematic reviews of interventions," 2011.

[15] C. Peifeng, "Clinical observation on primary hypertension treated with combination of traditional chinese and western medicines," Cardiovascular Disease Journal of Integrated Traditional Chinese and Western Medicine, vol. 10, no. 22, pp. 30-31, 2015.
[16] C. Xiaofei, Z. Baoxia, and Z. Baojun, "Clinical observation on 40 cases of hyper-yang of liver hypertension treated by tianma gouteng decoction combined with nifedipine controlled release tablets practical clinical," Journal of Integrated Traditional Chinese and Western Medicine, vol. 1, pp. 16-18, 2016.

[17] D. Tao, "Clinical Efficacy and safetyevaluation of tianma gouteng decoction combined with nifedipine sustained-release tablets in treating essential hypertension," Asia-Pacific Traditional Medicine, vol. 11, pp. 119-120, 2016.

[18] G. Yingke, "Clinical observation on tianma gouteng decoction adjuvant treatment hyper-yang of liver hypertension," Clinical Journal of Chinese Medicine, vol. 2, pp. 31-32, 2014.

[19] H. Jinbo, "Clinical efficacy observation of tianma gouteng decoction combined with nifedipine in the treatment hyperyang of liver hypertension," Journal of Clinical Medical Literature, vol. 19, 2015.

[20] K. Fan and T. Lili, "Efficacy observation of tianma gouteng decoction combined with nifedipine in the treatment hyperyang of liver hypertension," Nei Mongol Journal of Traditional Chinese Medicine, vol. 3, 2015.

[21] Z. Pan, "Clinical effect of tianma gouteng decoction combined with nifedipine sustained release tablets in the treatment of patients with hypertension," Strait Pharmaceutical Journal, vol. 31, no. 6, pp. 108-109, 2019.

[22] Q. Xiaofang, "Clinical observation of tianma gouteng decoction in the treatment of hyper-yang of liver hypertension," Journal of North Pharmacy, vol. 3, p. 59, 2016.

[23] X. Fu, "A randomized parallel controlled study of tianma gouteng decoction combined with nifedipine in the treatment of hypertension (hyperactivity of liver yang)," Journal of Practical Traditional Chinese Internal Medicine, vol. 32, no. 5, pp. 40-42, 2018.

[24] S. Caihong, "Therapeutic effect of tianma gouteng yin in the treatment of asthenic yin causing predominant yang in elderly hypertensive patients and its influence on quality of life," Chinese Journal of Clinical Rational Drug Use, vol. 31, pp. 44-46, 2017.

[25] J. Dai, "Analysis of therapeutic effect of tianma gouteng decoction combined with nifedipine sustained release tablets in the treatment of hypertension," World Latest Medicine Information, vol. 19, no. 11, p. 140, 2019.

[26] X. Yugao, "Clinical analysis of Tianma gouteng decoction in treatment of hyper-yang of liver hypertension," China Modern Medicine, vol. 15, pp. 114-116, 2017.

[27] Y. Peng, L. Yumei, L. Xiaowen, and W. Lu, "Clinical analysis of tianma gouteng decoction in treatment of hyper-yang of liver hypertension," China Continuing Medical Education, vol. 16, pp. 179-180, 2016.

[28] Z. Yingjun, "Observation of curative effect of tianma gouteng decoction on hyper-yang of liver hypertension," Practical Clinical Journal of Integrated Traditional Chinese and Western Medicine, vol. 3, pp. 14-15, 2015.

[29] Q. Xiaofang, "Observation of curative effect of tianma gouteng decoction in the treatment of hyper-yang of liver hypertension," Journal of North Pharmacy, vol. 3, p. 59, 2016.

[30] WHO, "A global brief on hypertension: silent, killer. global public health crisis," 2013.

[31] A. D. Lopez and C. C. J. L. Murray, "The global burden of disease, 1990-2020," Nature Medicine, vol. 4, no. 11, pp. 1241-1243, 1998.

[32] B. Williams, G. Mancia, W. Spiering et al., "2018 ESC/ESH guidelines for the management of arterial hypertension," Journal of Hypertension, vol. 36, no. 10, pp. 1953-2041, 2018.

[33] P. K. Whelton, R. M. Carey, W. S. Aronow et al., "ACC/AHA/ AAPA/ABC/ACPM/AGS/APhA/ASH/ASPC/NMA/PCNA 
guideline for the prevention, detection, evaluation, and management of high blood pressure in adults: executive summary," Journal of the American Society of Hypertension, vol. 12 , no. 8, p. $579,2017$.

[34] M. Ga, "Cost of poor blood pressure control in the UK: 62000 unnecessary deaths per year," Journal of Human Hypertension, vol. 17, pp. 455-457, 2003.

[35] F. Khoo and M. Mathur, "Severe resistant maternal hypotension following tocolysis with nifedipine," BMJ Case Reports, vol. 2014, 2014

[36] D. Yusuf, J. Christy, D. Owen, M. Ho, D. Li, and M. Fishman, "A case report of nifedipine-induced hepatitis with jaundice," BMC Research Notes, vol. 11, no. 1, p. 228, 2018.

[37] C. Biao, W. Shuyue, W. Yanning, and Z. Hui, "Case analysis of serious adverse reactions caused by nifedipine," China Pharmacist, vol. 8, pp. 1136-1137, 2009.

[38] N. Docheva, E. Slutsky, R. Sandelin, and J. Van Hook, “Atrial fibrillation as a rare complication of the use of nifedipine as a tocolytic agent: a case report and review of the literature," Case Reports in Obstetrics and Gynecology, vol. 2018, 2018.

[39] K. Shimamoto, M. Kimoto, Y. Matsuda, K. Asano, and M. Kajikawa, "Long-term safety and efficacy of high-dose controlled-release nifedipine ( $80 \mathrm{mg}$ per day) in Japanese patients with essential hypertension," Hypertension Research, vol. 38, no. 10, pp. 695-700, 2015.

[40] X. Guicheng and L. Kun, "Chinese medicine treatment of hypertension,” People's Military Medical Press, vol. 10, p. 1, 2012.

[41] L. Baoguo, B. Baoyin, S. Haifa et al., "Tianma gouteng decoction effect on asthenic yin causing predominant yang hypertension patients at plasma ET," PRA and AII Chinese General Practice, vol. 10, no. 3, pp. 190-191, 2000.

[42] L. ZhenYan, L. Wei, Y. YongPing et al., "Effect of tianma gouteng decoction on differential protein expression in hypothalamus," Chinese Journal of Tissue Engineering Research, vol. 47, pp. 58-61, 2006.

[43] Y. Fengmei, S. Liexing, and X. Ruijun, "Pharmacological research and clinical application of tianma gouteng decoction forum on traditional chinese medicine," China Health Industry, vol. 3, pp. 51-53, 2008.

[44] S. Qingyang, X. Mingfu, and W. Xu, "Clinical study on hypertension treated by tianma gouteng decoction shandong," Journal of Traditional Chinese Medicine, vol. 6, pp. 336-338, 2005.

[45] X. Shihu, "Identification and pharmacological effects of gastrodia," China Health Industry, vol. 21, pp. 167-169, 2016.

[46] L. Feng, A. Manavalan, M. Mishra, S. K. Sze, J.-M. Hu, and K. Heese, "Tianma modulates blood vessel tonicity," The Open Biochemistry Journal, vol. 6, pp. 56-65, 2012.

[47] A. Liu, Y. Chu, X. Wang et al., "Serum metabolomics study based on LC-MS and antihypertensive effect of on spontaneously hypertensive rats," Evidence-Based Complementary and Alternative Medicine, vol. 2018, 2018.

[48] X. Linhu, W. Huadong, S. Dandong, P. Dong, and D. Boping, "Research progress on the pharmacological effects of rhynchophylline on cardiovascular system," Science \& Technology Vision, vol. 1, pp. 65-66, 2017. 